\title{
A meta-analysis of the diagnostic accuracy of dengue virus-specific IgA antibody-based tests for detection of dengue infection
}

\author{
K. ALAGARASU ${ }^{1 *}$, A. M. WALIMBE ${ }^{2}$, S. M. JADHAV ${ }^{2}$ \\ AND A. R. DEOSHATWAR ${ }^{3}$ \\ ${ }^{1}$ Dengue/Chikungunya Group, National Institute of Virology, Pune, Maharashtra, India \\ ${ }^{2}$ Bioinformatics and Data Management Group, National Institute of Virology, Pune, Maharashtra, India \\ ${ }^{3}$ Epidemiology Group, National Institute of Virology, Pune, Maharashtra, India
}

Received 16 February 2015; Final revision 27 July 2015; Accepted 29 July 2015;

first published online 20 August 2015

\section{SUMMARY}

Immunoglobulin A (IgA)-based tests have been evaluated in different studies for their utility in diagnosing dengue infections. In most of the studies, the results were inconclusive because of a small sample size. Hence, a meta-analysis involving nine studies with 2096 samples was performed to assess the diagnostic accuracy of IgA-based tests in diagnosing dengue infections. The analysis was conducted using Meta-Disc software. The results revealed that IgA-based tests had an overall sensitivity, specificity, diagnostic odds ratio, and positive and negative likelihood ratios of $73 \cdot 9 \%, 95 \cdot 2 \%, 66 \cdot 7,22 \cdot 0$ and $0 \cdot 25$, respectively. Significant heterogeneity was observed between the studies. The type of test, infection status and day of sample collection influenced the diagnostic accuracy. The IgA-based diagnostic tests showed a greater accuracy when the samples were collected 4 days after onset of symptoms and for secondary infections. The results suggested that IgA-based tests had a moderate level of accuracy and are diagnostic of the disease. However, negative results cannot be used alone for dengue diagnosis. More prospective studies comparing the diagnostic accuracy of combinations of antigen-based tests with either IgA or IgM are needed and might be useful for suggesting the best strategy for dengue diagnosis.

Key words: Dengue fever, dengue haemorrhagic fever, immunology, meta-analysis, IgA.

\section{INTRODUCTION}

Dengue, caused by four serotypes of dengue virus (DENV), poses a major threat to public health systems in tropical and subtropical countries and is an emerging threat to countries where it has not yet been reported. Disease burden studies from South East Asian countries have estimated 209 million dengue episodes and 5906 deaths during 2001-2010 [1].

\footnotetext{
* Author for correspondence: Dr K. Alagarasu, Dengue/ Chikungunya Group, National Institute of Virology, 20A Dr Ambedkar Road, Pune, Maharshtra, India.

(Email: alagarasu@gmail.com)
}

Dengue is a complex disease with diverse range of clinical manifestations ranging from mild forms (undifferentiated fever and dengue fever) to severe forms of the disease (dengue haemorrhagic fever, dengue shock syndrome and expanded dengue syndrome/ isolated organopathy/unusual manifestations) [2]. During the febrile phase, the symptoms mimic those caused by a wide spectrum of viral, bacterial and protozoal infections, making diagnosis difficult.

Rapid and accurate diagnosis is essential for effective clinical management of dengue cases. Nucleic acid-based tests offer the best opportunity for early diagnosis of dengue but are expensive and not available in resource-limited settings. Non-structural 
protein 1 (NS1) antigen-based enzyme linked immunosorbent assays (ELISAs) and rapid immunochromatographic tests (ICTs) offer a better alternative for nucleic acid-based tests in early diagnosis of dengue. These tests are less expensive and are used even in primary health centres. However, recent studies have indicated that the sensitivity of NS1 antigen-based tests are lower in secondary infections and in DENV-4 serotype infections [3-5].

The anti-DENV IgM capture ELISA (MACELISA) test is widely used for probable diagnosis of dengue in resource-limited settings. IgM antibodies against DENV appear 3-5 days after the onset of symptoms and can persist for 2-3 months. The sensitivity for MAC-ELISA is above $90 \%$ and the specificity is $98 \%$ for samples collected after 5 days of onset of symptoms [6]. However, IgM levels are lower in secondary infections and about $28 \%$ of secondary infections were not detected by MAC-ELISA [7]. Anti-DENV IgG-based ELISAs have been utilized for discriminating between primary and secondary infections as well as to rule out past infections [6].

Based on the observation that the anti-DENV IgA antibodies appear at the same time as IgM antibodies and persist for shorter periods compared to IgM, Talarmin et al. tested an IgA capture ELISA (AACELISA) for its utility in diagnosing dengue infections [8]. An antigen capture anti-DENV IgA ELISA (ACA-ELISA) for diagnosis of dengue has been reported with higher sensitivity and specificity [9]. An ICT, which detects anti-DENV IgA antibodies was evaluated for its utility in diagnosing dengue in various countries [10]. Recently, a commercial dengue IgA capture ELISA kit was made available and tested for its utility in dengue diagnosis [11]. Most of these studies have been conducted with small sample sizes and a meta-analysis may provide more reliable estimates about the diagnostic accuracy of IgA-based tests. In the present study, we undertook a systematic review and meta-analysis of the diagnostic accuracy of IgA-based tests for diagnosis of dengue.

\section{MATERIALS AND METHODS}

\section{Search criteria and study selection}

The articles used for the meta-analysis were searched in NCBI Pubmed using the following search criteria: (immunoglobulin A OR IgA) AND (dengue OR dengue fever OR dengue hemorrhagic fever OR dengue shock syndrome) AND diagnosis AND sensitivity. Articles published up to March 2015 were included. Additional studies were included by searching the references of the articles derived from Pubmed and also by searching citations of articles in Google Scholar. Inclusion criteria were: (1) studies that evaluated the sensitivity and specificity of any IgA-based assay against the gold standard assay for diagnosis using serum samples, and (2) studies in English language. The following types of studies were excluded: (1) studies with incomplete data for calculating true positives and true negatives, (2) conference abstracts, commentaries, review articles and editorials, and (3) studies which used samples other than serum (capillary blood, saliva, urine). The criteria suggested by World Health Organization for diagnosis of confirmed dengue were considered as the gold standard [12]. The samples met any one of the following criteria:

(1) Samples which were positive either by virus isolation or by reverse transcriptase-polymerase chain reaction (RT-PCR)/real-time RT-PCR.

(2) Samples which showed IgM seroconversion in paired sera.

(3) Samples which showed IgG seroconversion or increase in $\mathrm{IgG}$ titre in paired sera.

The above criteria were considered as confirmation for dengue samples.

The literature search was performed independently by two reviewers.

\section{Data extraction}

The following data were extracted from the articles selected for meta-analysis: authors, year of publication, index test used, reference standards used, sample size, number of dengue cases, number of non-dengue cases, day of sample collection, whether primary or secondary infection, serotypes, and the number of true positives, true negatives, false positives and false negatives. If a study contained multiple sample sets, only the sample set which was tested by gold standard reference assays were included. The sample sets with a single sample tested by IgM or IgG or NS1 Ag ELISAs were excluded from the analysis. The following criteria were used for subgroup analysis: type of index test used, primary/secondary infection, and day of sample collection. The data extraction was performed independently by two reviewers and disagreements were resolved by discussion.

\section{Quality assessment}

The quality assessment of the selected studies was performed by two reviewers independently using the 
QUADAS 2 tool [13] and discrepancies were resolved by discussion. The risk of bias was assessed in four key domains covering patient selection, index test, reference standard, and flow of patients through the study. The risk of bias in each domain was judged in terms of 'low', 'high' and 'unclear'. The first three domains were also assessed in terms of concerns regarding applicability. Risk of bias and the applicability-concerns graph was generated using Review Manager 5.3 software [14].

\section{Statistical analysis}

A meta-analysis was performed using Meta-Disc software version 1.4 [15]. The sensitivity, specificity, diagnostic odds ratio (dOR), positive likelihood ratio $(\mathrm{LR}+)$ and negative likelihood ratio (LR-) were calculated for each study and the pooled estimates of these parameters were obtained using a random-effects model (DerSimonianLaird method). The Cochran $Q$ test was used to test the homogeneity of likelihood ratios and diagnostic odds ratios across the studies. The effect of heterogeneity for each parameter was provided by the $I^{2}$ statistic, which described the percentage of total variation across the studies due to heterogeneity. The probable reasons for heterogeneity were investigated by stratifying the data on the basis of type of index test, primary/secondary infection and the day of sample collection. A meta-regression analysis was performed to discover whether the year of publication and sample size affected the diagnostic accuracy of IgA-based tests. Fagan plot analysis was performed in order to estimate how much the result of a diagnostic test changes the probability that a patient has the disease. This analysis was based on the positive and negative likelihood ratios estimated by the meta-analysis and was performed for three pre-test probabilities of $25 \%, 50 \%$ and $75 \%$ [16]. Publication bias was assessed by Deeks' funnel plot asymmetry test [17]. Both Fagan plot analysis and Deeks' funnel plot asymmetry test were performed using Stata version 13 (StataCorp., USA).

\section{Ethical approval}

This work does not contain any studies with human participants or animals performed by any of the authors.

\section{RESULTS}

\section{Search results}

Using the search criteria in Pubmed/Medline, 14 studies were obtained. Eleven articles which evaluated the diagnostic accuracy of IgA-based test for diagnosis of dengue were shortlisted. The reference lists and citations of the shortlisted articles were also searched for additional articles leading to the addition of a further four articles. After applying the inclusion and exclusion criteria, nine articles were selected for the final meta-analysis (Table 1). The remaining six articles were excluded for reasons mentioned in Table 2. Of these, one article was excluded because denguenegative samples were not included in the study while another article was excluded because the data was based on capillary blood samples [18, 20]. Four other articles were excluded due to the use of inappropriate gold standard assays [19, 25, 27, 28].

\section{Characteristics of the studies used for analysis}

In total, nine articles were used for the meta-analysis. Three studies used AAC-ELISA, while one study used ACA-ELISA [8, 9, 21, 22]. One study used a commercially available ELISA (Platelia Dengue IgA Capture, Bio-Rad, USA) [11]. An immunofluorescence assay was used in one study [23]. A commercially available ICT (ASSURE $^{\circledR}$ Dengue IgA Rapid test, MP Biomedicals, USA) was used by three studies [10, 24, 26]. In total, 2096 samples were used for the meta-analysis. Only a subset of samples which were tested against the gold standard reference assay was included from four studies [8, 10, 21, 24] (Table 1).

The quality of the studies in terms of the risk of bias was assessed in four key domains covering patient selection, index test, reference standard, and flow of patients through the study and the timing of the index test and reference standards. The summary of the results of quality assessment using the QUADAS 2 tool is given in Figure 1. Three $(33 \cdot 3 \%)$ studies had a high risk of bias with regard to selection of samples. All three studies had included healthy controls as dengue-negative subjects $[21,23,26]$. Six $(66 \cdot 7 \%)$ studies had an unclear risk of bias with regard to the performance of index test. In all of these six studies, it was unclear whether the index test was performed without the knowledge of results of the reference test $[8,10,21-23,26]$. One $(11 \cdot 1 \%)$ study had a high risk of bias with regard to the performance of the index test [9]. In this study, the reference test results were not blinded to the personnel who performed the index test. All studies had a lower risk of bias with regard to the conduct of the reference test and the flow and timing of samples. There was no or low risk of concern regarding the applicability in terms of patient selection, index test and reference test. 
Table 1. Characteristics of the studies used for meta-analysis of diagnostic accuracy of IgA in detection of dengue

\begin{tabular}{|c|c|c|c|c|c|}
\hline $\begin{array}{l}\text { Author, year } \\
\text { [ref.] }\end{array}$ & $\begin{array}{l}\text { Sample } \\
\text { size }\end{array}$ & Index test & Reference test & Sample timing & Infection status \\
\hline $\begin{array}{l}\text { Talarmin et al. } \\
1998[8]^{*}\end{array}$ & 280 & $\begin{array}{l}\text { IgA capture } \\
\text { ELISA }\end{array}$ & $\begin{array}{l}\text { Seroconversion, fourfold rise in } \\
\text { hemagglutination inhibition titre, } \\
\text { virus isolation and RT-PCR }\end{array}$ & 1-29 days & $\begin{array}{l}\text { Not known for } \\
\text { many samples }\end{array}$ \\
\hline $\begin{array}{l}\text { Groen et al. } \\
1999[23]\end{array}$ & 120 & IFA & Seroconversion in paired sera & 5-8 days & Primary/secondary \\
\hline $\begin{array}{l}\text { Balmaseda et al. } \\
2003[21]^{*}\end{array}$ & 201 & $\begin{array}{l}\text { IgA capture } \\
\text { ELISA }\end{array}$ & $\begin{array}{l}\text { IgM/RT-PCR/inhibition ELISA in } \\
\text { single samples, seroconversion or } \\
\text { inhibition ELISA titre rise in paired } \\
\text { sera }\end{array}$ & $0-7$ days & Not known \\
\hline $\begin{array}{l}\text { Balmaseda et al. } \\
2008 \text { [22] }\end{array}$ & 356 & $\begin{array}{l}\text { IgA capture } \\
\text { ELISA }\end{array}$ & $\begin{array}{l}\text { Seroconversion, fourfold rise in } \\
\text { inhibition ELISA titre, RT-PCR } \\
\text { and virus isolation }\end{array}$ & $0-4$ days & $\begin{array}{l}\text { Primary/secondary } \\
\text { data given (exact } \\
\text { number not } \\
\text { known) }\end{array}$ \\
\hline $\begin{array}{l}\text { Ahmed et al. } \\
2010[24]^{*}\end{array}$ & 204 & ICT & $\begin{array}{l}\text { Reference ELISAs - IgM, IgG and } \\
\text { NS1 Ag in paired sera }\end{array}$ & $\begin{array}{l}\text { Acute phase } \\
\text { sera (days not } \\
\text { mentioned) }\end{array}$ & Primary/secondary \\
\hline $\begin{array}{l}\text { Tan et al. } 2011 \\
{[10]^{*}}\end{array}$ & 382 & ICT & $\begin{array}{l}\text { Reference ELISAs - IgM, IgG and } \\
\text { NS1 Ag, RT-PCR, seroconversion } \\
\text { in paired sera using RT-PCR and } \\
\text { IgM ELISA }\end{array}$ & $\begin{array}{l}\text { Acute phase } \\
\text { sera (days not } \\
\text { mentioned) }\end{array}$ & Primary/secondary \\
\hline $\begin{array}{l}\text { Yap et al. } 2011 \\
\text { [9] }\end{array}$ & 144 & $\begin{array}{l}\text { Antigen } \\
\text { capture anti } \\
\text { DENV IgA } \\
\text { ELISA }\end{array}$ & $\begin{array}{l}\text { Seroconversion in paired sera using } \\
\text { RT-PCR and IgM ELISA }\end{array}$ & 1-8 days & Primary/secondary \\
\hline $\begin{array}{l}\text { de la cruz } \\
\text { Hernández } \\
\text { et al. } 2012 \text { [26] }\end{array}$ & 225 & ICT & $\begin{array}{l}\text { NS1 Ag ELISA and real-time RT- } \\
\text { PCR }\end{array}$ & $0-5$ days & Primay/secondary \\
\hline $\begin{array}{l}\text { De Decker } \text { et al. } \\
2015 \text { [11] }\end{array}$ & 184 & $\begin{array}{l}\text { Platelia } \\
\text { Dengue IgA } \\
\text { Capture, } \\
\text { Bio-Rad }\end{array}$ & Virus isolation, RT-PCR & $3-15$ days & Primary/secondary \\
\hline
\end{tabular}

IFA, Immunoflourescence assay; ICT, immunochromatographic test.

* Only a subset of samples were used.

Table 2. Studies that were excluded from meta-analysis and reasons for exclusion

\begin{tabular}{|c|c|}
\hline Author, year [ref.] & Reason for exclusion \\
\hline Nawa et al. 2005 [19] & Inappropriate gold standard \\
\hline Nawa et al. 2006 [18] & $\begin{array}{l}\text { Dengue negative samples were not } \\
\text { studied }\end{array}$ \\
\hline $\begin{array}{l}\text { Sharmin et al. } 2012 \\
{[25]}\end{array}$ & Inappropriate gold standard \\
\hline Hasan et al. 2013 & Inappropriate gold standard \\
\hline Naz et al. 2014 [28] & Inappropriate gold standard \\
\hline $\begin{array}{l}\text { Matheus et al. } 2014 \\
\text { [20] }\end{array}$ & $\begin{array}{l}\text { Data was based on capillary blood } \\
\text { samples }\end{array}$ \\
\hline
\end{tabular}

\section{Overall diagnostic accuracy of $\operatorname{IgA}$-based test for diagnosis of dengue infection}

When all the nine studies were analysed in metaanalysis, the sensitivity values (proportion of testpositives in subjects with disease) ranged from $47 \cdot 6 \%$ to $93.0 \%$ and the pooled estimate was $73.9 \%$ [95\% confidence interval (CI) 71·6-76.0] (Fig. 2a). The specificity values (proportion of test-negatives in subjects without disease) ranged from $86.0 \%$ to $100 \%$ and the pooled estimate was $95 \cdot 2 \%(95 \%$ CI $93 \cdot 0-96 \cdot 9)$ (Fig. 2b).

The LR+ (number of times a positive index test result more likely in the diseased group compared to the non-diseased group) ranged from 6.66 to 107.65 with a pooled estimate of 22.0 (95\% CI 8.4-37.3) (Fig. 3a). The LR- (number of times a negative 


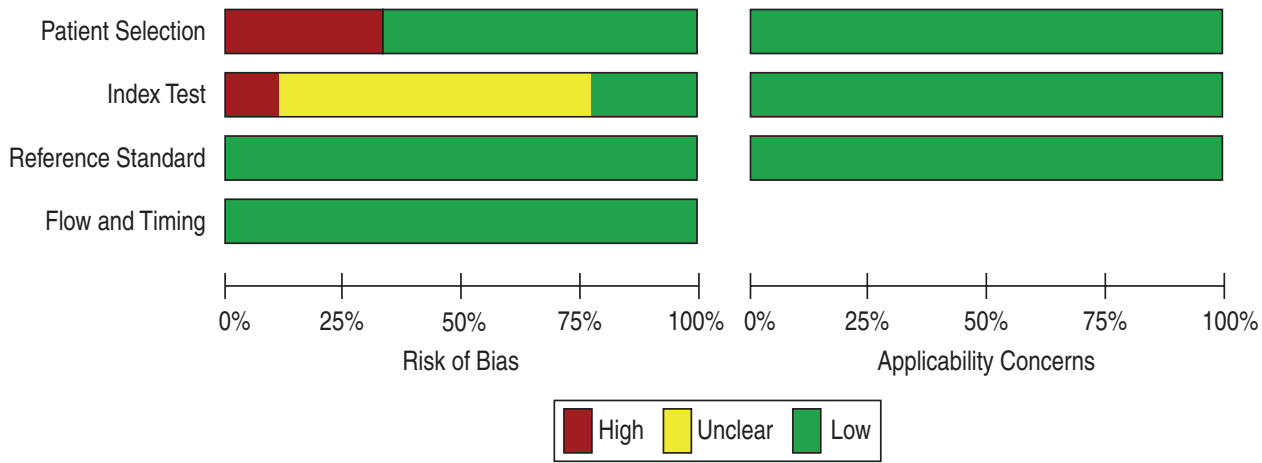

Fig. 1. Risk of bias and applicability-concerns graph: a review of authors' judgements about each domain presented as percentages across the included studies.

(a)

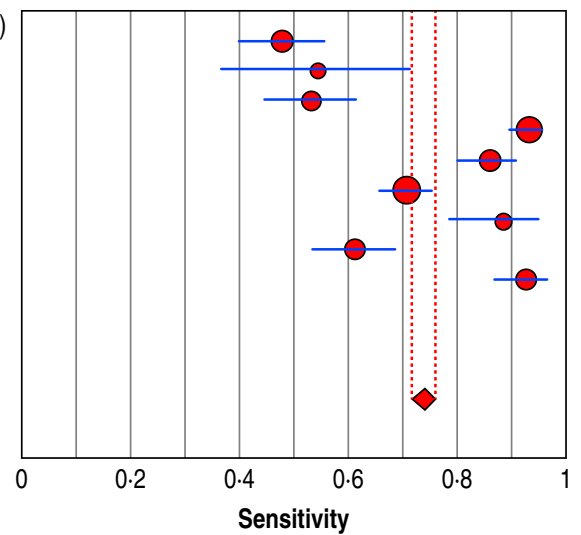

(b)

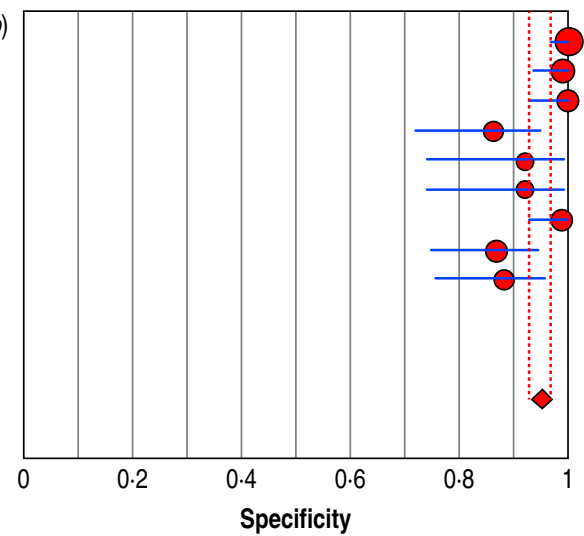

Sensitivity $(95 \% \mathrm{Cl})$

Talarmin et al. (1998) [8] Groen et al. (1999) [23]

Balmaseda et al. (2003) [21]

Balmaseda et al. (2008) [22]

Ahmed et al. (2010) [24]

Tan et al. (2011) [10]

Yap et al. (2011) [9]

De Decker et al. (2015) [11]

Pooled Sensitivity $=0.74(0.72$ to 0.76$)$

Chi-square $=235.42 ; d f=8(p=0.0000)$

Inconsistency (I-square) $=96.6 \%$

\section{Specificity $(95 \% \mathrm{Cl})$}

Talarmin et al. (1998) [8]

Groen et al. (1999) [23]

Balmaseda et al. (2003) [21]

Balmaseda et al. (2008) [22]

Ahmed et al. (2010) [24]

Tan et al. (2011) [10]

Yap et al. (2011) [9]

De la Cruz Hernández et al. (2012) [26] $0.87 \quad(0.75-0.95)$

De Decker et al. (2015) [11]

$0.88 \quad(0.76-0.95)$

Pooled Specificity $=0.95(0.93$ to 0.97$)$

Chi-square $=38.14 ; d f=8(p=0.0000)$

Inconsistency (I-square) $=79.0 \%$

Fig. 2. Forest plots for $(a)$ sensitivity and $(b)$ specificity of IgA-based tests. Forest plot for sensitivity or specificity of each individual study as well as the pooled estimate are represented by solid circles and the horizontal lines represent $95 \%$ confidence intervals (CI).

index test result less likely in the diseased group compared to the non-diseased group) ranged from 0.08 to 0.53 with a pooled estimate of $0.25(95 \%$ CI $0.17-$ 0.36) (Fig. 3b).

The dOR (odds of obtaining a positive test result in a diseased individual compared to a non-diseased individual) ranged from $10 \cdot 30$ to $564 \cdot 25$ with a pooled estimate of $66 \cdot 77$ (95\% CI 28.70-155.33) (Fig. 4).

The heterogeneity presented as $I^{2}$ values (i.e. quantifies the variation in excess of that due to chance across studies) were significantly higher than 50 for all the parameters studied (Figs 2-4). 
Positive LR (95\% Cl)
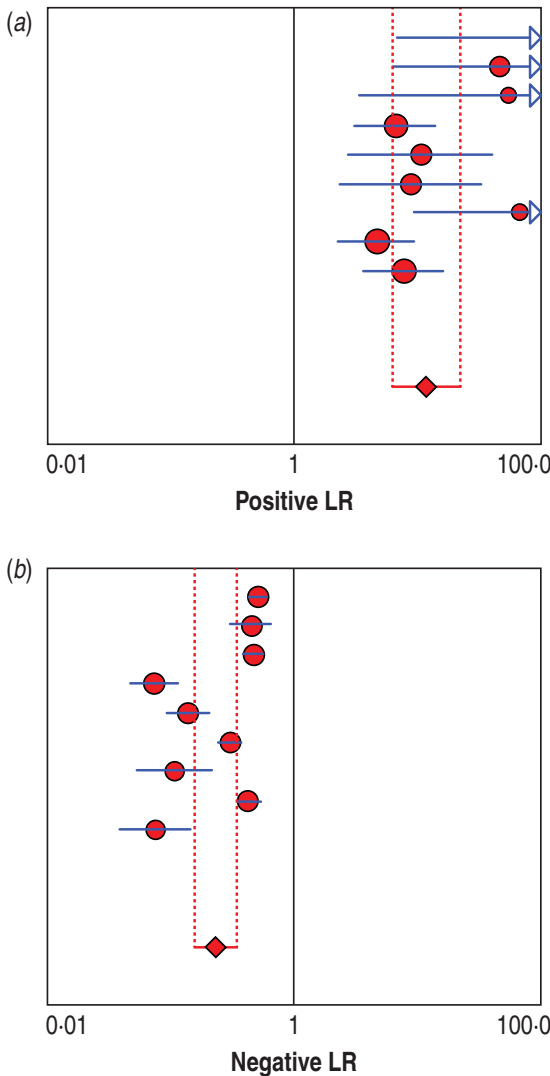

Talarmin et al. (1998) [8]

Groen et al. (1999) [23]

Balmaseda et al. (2003) [21]

Balmaseda et al. (2008) [22]

Ahmed et al. (2010) [24]

Tan et al. (2011) [10]

Yap et al. (2011) [9]

De Decker et al. (2015) [11]

Random Effects Model

Pooled Positive LR $=22.0$ (8.4 to 37.3)

Cochran- $Q=18.22 ; d f=8(p=0.0196)$

Inconsistency (l-square) $=56.1 \%$

Tau-squared $=0.4421$

Negative LR (95\% Cl)

Talarmin et al. (1998) [8] $\quad 0.53 \quad(0.46-0.61)$

Groen et al. (1999) [23] $0.46 \quad(0.32-0.66)$

Balmaseda et al. (2003) [21] $\quad 0.48 \quad(0.40-0.56)$

Balmaseda et al. (2008) [22] $\quad 0.08 \quad(0.05-0.12)$

Ahmed et al. (2010) [24] $\quad 0.15 \quad(0.10-0.22)$

$\begin{array}{lll}\text { Tan et al. (2011) [10] } & 0.32 & (0.26-0.39)\end{array}$

Yap et al. (2011) [9] $\quad 0.12 \quad(0.06-0.23)$

De la Cruz Hernández et al. (2012) [26] $0.45 \quad(0.36-0.56)$

$\begin{array}{lll}\text { De Decker et al. (2015) [11] } & 0.08 & (0.05-0.16)\end{array}$

Random Effects Model

Pooled Negative LR $=0.25$ (0.17 to 0.36$)$

Cochran- $Q=158.29 ; d f=8(p=0.0000)$

Inconsistency (I-square) $=94.9 \%$

Tau-squared $=0.3154$

Fig. 3. Forest plots for (a) positive likelihood ratio (LR) and (b) negative LR of IgA-based tests. LRs of each individual study and the pooled estimate are represented by solid circles and the horizontal lines represent $95 \%$ confidence intervals (CI).

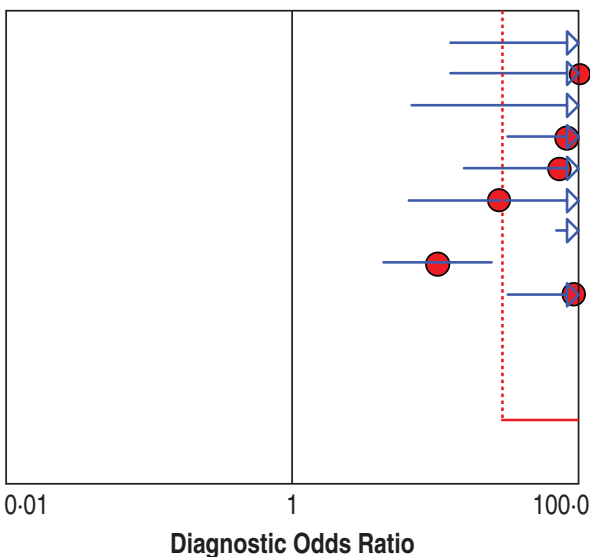

Talarmin et al. (1998) [8]

Groen et al. (1999) [23]

Balmaseda et al. (2003) [21]

Balmaseda et al. (2008) [22]

Ahmed et al. (2010) [24]

Tan et al. (2011) [10]

Yap et al. (2011) [9]

De la Cruz Hernandez et al. (2012) [26] $10.30 \quad(4.39-24.15)$

De Decker et al. (2015) [11]

Random Effects Model

Pooled Diagnostic Odds Ratio $=66.77$ (28.70 to 155.33 )

Cochran- $Q=23.12 ; d f=8(p=0.0032)$

Inconsistency (I-square) $=65.4 \%$

Tau-squared $=0.9688$

Fig. 4. Forest plot of diagnostic odds ratio of IgA-based tests. The diagnostic odds ratios of each individual study and the pooled estimate are represented by solid circles and the horizontal lines represent $95 \%$ confidence intervals (CI).

Influence of the type of test employed, day of sample collection and infection status on the diagnostic accuracy of IgA-based tests in diagnosing dengue

Since the heterogeneity between the studies was significant, a subgroup analysis was performed based on the type of test employed, day of sample collection and infection status to identify the source of heterogeneity.

Five studies had used an ELISA-based test while three studies had used an ICT-based test. The subgroup analysis, based on the type of the test employed, 
revealed that the ELISA-based test had sensitivity and specificity of $76 \%$ (95\% CI 73-79) and 96\% (95\% CI 94-98), respectively. The dOR was 108.42 (95\% CI 57.07-205.95) while LR+ was $17 \cdot 11(95 \%$ CI $5 \cdot 87-$ 49.86) and LR- was $0 \cdot 19$ (95\% CI $0 \cdot 09-0 \cdot 40)$. Heterogeneity was not observed between the studies for dOR $\left(I^{2}=0\right)$. For ICT-based test, the sensitivity, specificity, dOR, LR+ and LR-were 72\% (95\% CI 69-75), $89 \%$ (95\% CI 82-95), $23 \cdot 82$ (95\% CI 7.44-76.22), 6.03 (95\% CI $3 \cdot 43-10 \cdot 57)$, and $0 \cdot 29$ (95\% CI 0.17-0.47), respectively. Heterogeneity was not observed between the studies for specificity and $\mathrm{LR}+\left(I^{2}=0\right)$. A moderate heterogeneity was observed for dOR $\left(I^{2}=61 \cdot 3 \%\right)$ (Table 3$)$.

Complete data for the day of sample collection after the onset of symptoms was available for four studies. Based on the available data, samples were categorized into two groups, samples collected up to 4 days before and samples collected 4 days after symptom onset. For samples which were collected up to 4 days before, sensitivity, specificity, dOR, LR+ and LR- were 78\% (95\% CI $74-81)$, 92\% (95\% CI 88-96), 35.91 (95\% CI $8.78-$ 146.91), 6.99 (95\% CI 3.39-14.43), and 0.28 (95\% CI $0 \cdot 14-0 \cdot 56)$, respectively. The heterogeneity was significant between the studies for all parameters except for $\mathrm{LR}+\left(I^{2}=45 \cdot 4 \%\right)$. For samples collected 4 days after onset of symptoms, the sensitivity, specificity, dOR, LR+ and LR- were 86\% (95\% CI 78-92), 93\% (95\% CI $88-97), 85.68$ (95\% CI 15.51-473.25), 13.56 (95\% CI 3.01-61·01), and $0 \cdot 153(95 \%$ CI $0 \cdot 1-024)$, respectively. Heterogeneity between the studies was not observed for sensitivity and LR- $\left(I^{2}=0\right)$ and a moderate heterogeneity was observed for dOR $\left(I^{2}=65 \cdot 8 \%\right)$ (Table 3 ).

Six studies had complete data on the infection status and samples were categorized into those from primary or secondary infections. For samples from primary dengue infection, the sensitivity, specificity, dOR, LR+ and LR- were 68\% (95\% CI 63-73), 96\% (95\% CI 94-98), 56.44 (95\% CI 14.07-226.31), 14.26 (95\% CI 5.38$37 \cdot 83)$, and $0 \cdot 32(95 \% \mathrm{CI} 0 \cdot 18-0 \cdot 57)$, respectively. In samples from secondary infections, the sensitivity, specificity, dOR, LR+ and LR- were $92 \%$ (95\% CI 89-94), 96\% (95\% CI 94-98), 321.65 (95\% CI 59.84-1728.9), 20.09 (95\% CI 7.13-56.64), and 0.09 (95\% CI 0.03-0.26), respectively. Heterogeneity was significant between the studies in both the groups $\left(I^{2}>75 \%\right)$ (Table 3$)$.

\section{Influence of the year of publication and sample size on the diagnostic accuracy of IgA-based tests for detecting dengue}

Further, to discover whether the year of publication and sample size affected the diagnostic accuracy of IgA-based tests, a meta-regression analysis was performed. For the year of publication, the studies were categorized into those published on or before 2007 and after 2007 (the cutoff was chosen on the basis that the first study on use of IgA for dengue diagnosis was published in 1998 and hence the median year between 1998 and 2015 was considered). To study the influence of sample size, mean study sample size was calculated to be 234 and the studies were categorized into those with sample size $\leqslant 234$ and those with sample size $>234$. A meta-regression analysis revealed that the year of publication $(P=0.74)$ and the sample size $(P=0 \cdot 28)$ had no influence on the diagnostic accuracy of IgA-based tests for detecting dengue.

Fagan plot analysis to estimate the post-test probability based on simulation of an environment with different pre-test probability

To estimate the post-test probability associated with IgA-based diagnostic tests in an environment with different prevalence rates of dengue disease, Fagan plot analysis was performed for three pre-test probabilities: $25 \%, 50 \%$ and $75 \%$. The analysis revealed that diagnosis using an IgA-based test would be incorrect in $8 \%, 20 \%$ and $43 \%$ of dengue cases when the prevalence rates were $0 \cdot 25,0.5$ and $0 \cdot 75$, respectively. It further indicated that diagnosis would be correct in $88 \%$, $96 \%$ and $99 \%$ of dengue cases when the prevalence rates were $0 \cdot 25,0 \cdot 5$ and $0 \cdot 75$, respectively (Fig. 5).

\section{Assessment of publication bias}

Publication bias was assessed using Deeks' funnel plot asymmetry test. The results revealed that the funnel plot was symmetrical suggesting that there was no publication bias $(P=0.44)$ (Fig. 6).

\section{DISCUSSION}

The results of the meta-analysis showed that using IgA-based assays to diagnose dengue had an overall sensitivity of $74 \%$ and a specificity of $95 \%$. The overall dOR, $\mathrm{LR}+$ and LR-were $66 \cdot 8,22 \cdot 0$ and $0 \cdot 25$, respectively. The results suggest that patients with dengue had a 22 -fold higher chance of being positive for IgA, while if the patient was negative for IgA, the probability of the patient having dengue was $25 \%$. Thus, the overall dOR, LR+ and LRresults indicate that IgA-based tests have a moderate level of accuracy and that they are diagnostic of only disease. The negative results cannot be used alone for diagnosis of dengue. 
Table 3. Subgroup analysis of diagnostic accuracy of IgA for detection of dengue based on the type of index test, sample timing and infection status

\begin{tabular}{|c|c|c|c|c|c|}
\hline $\begin{array}{l}\text { Subgroups and } \\
\text { heterogeneity between } \\
\text { studies for each subgroup }\end{array}$ & $\begin{array}{l}\text { Sensitivity } \\
(95 \% \mathrm{CI})\end{array}$ & Specificity $(95 \% \mathrm{CI})$ & $\begin{array}{l}\text { Diagnostic odds ratio } \\
(95 \% \mathrm{CI})\end{array}$ & $\begin{array}{l}\text { Positive likelihood } \\
\text { ratio }(95 \% \mathrm{CI})\end{array}$ & $\begin{array}{l}\text { Negative likelihood } \\
\text { ratio }(95 \% \mathrm{CI})\end{array}$ \\
\hline \multicolumn{6}{|l|}{ Type of index test used } \\
\hline $\begin{array}{l}\text { ELISA-based IgA tests } \\
(n=1165)\end{array}$ & $0 \cdot 76(0 \cdot 73-0 \cdot 79)$ & $0.96(0.94-0.98)$ & $108 \cdot 42(57 \cdot 07-205 \cdot 95)$ & $17 \cdot 11(5 \cdot 87-49 \cdot 86)$ & $0 \cdot 19(0 \cdot 09-0 \cdot 40)$ \\
\hline$I^{2}$ & $98 \cdot 0$ & $85 \cdot 5 \%$ & $0 \% *$ & $66 \cdot 3 \%$ & $97 \cdot 2 \%$ \\
\hline $\begin{array}{l}\text { ICT-based IgA tests } \\
(n=811)\end{array}$ & $0 \cdot 72(0 \cdot 69-0 \cdot 75)$ & $0 \cdot 89(0 \cdot 82-0.95)$ & $23 \cdot 82(7 \cdot 44-76 \cdot 22)$ & $6 \cdot 03(3 \cdot 43-10 \cdot 57)$ & $0 \cdot 29(0 \cdot 17-0 \cdot 47)$ \\
\hline$I^{2}$ & $93 \cdot 3 \%$ & $0 \% *$ & $61 \cdot 3 \% *$ & $0 \% *$ & $91 \cdot 6 \%$ \\
\hline \multicolumn{6}{|l|}{ Sample timing } \\
\hline$\leqslant 4$ days $(n=764)$ & $0 \cdot 78(0 \cdot 74-0 \cdot 81)$ & $0.92(0 \cdot 88-0 \cdot 96)$ & $35 \cdot 91(8 \cdot 78-146 \cdot 91)$ & $6 \cdot 99(3 \cdot 39-14 \cdot 43)^{*}$ & $0 \cdot 26(0 \cdot 14-0 \cdot 56)$ \\
\hline$I^{2}$ & $97 \cdot 0 \%$ & $81 \cdot 1 \%$ & $77 \cdot 9 \%$ & $45 \cdot 4 \%$ & $95 \cdot 0 \%$ \\
\hline$>4$ days $(n=1324)$ & $0 \cdot 86(0 \cdot 78-0 \cdot 92)$ & $0.93(0.88-0.97)$ & $85 \cdot 68(15 \cdot 51-473 \cdot 25)$ & $13 \cdot 56(3 \cdot 01-61 \cdot 01)$ & $0 \cdot 153(0 \cdot 1-024)$ \\
\hline$I^{2}$ & $0 \% *$ & $74 \cdot 9 \%$ & $65 \cdot 8 \% *$ & $75 \cdot 9 \%$ & $0 \% *$ \\
\hline \multicolumn{6}{|l|}{ Infection status } \\
\hline Primary $(n=865)$ & $0 \cdot 68(0 \cdot 63-0 \cdot 73)$ & $0.96(0.94-0.98)$ & $56 \cdot 44(14 \cdot 07-226 \cdot 31)$ & $14 \cdot 26(5 \cdot 38-37 \cdot 83)$ & $0 \cdot 32(0 \cdot 18-0 \cdot 57)$ \\
\hline$I^{2}$ & $84 \cdot 5 \%$ & $81 \cdot 5 \%$ & $79 \cdot 0 \%$ & $73 \cdot 8 \%$ & $90 \cdot 6 \%$ \\
\hline Secondary $(n=896)$ & $0 \cdot 92(0 \cdot 89-0 \cdot 94)$ & $0.96(0.94-0.98)$ & $321 \cdot 65(59 \cdot 84-1728 \cdot 9)$ & $20 \cdot 09(7 \cdot 13-56 \cdot 64)$ & $0.09(0.03-0 \cdot 26)$ \\
\hline$I^{2}$ & $88 \cdot 9 \%$ & $81 \cdot 5 \%$ & $78 \cdot 1 \%$ & $79 \cdot 9 \%$ & $88 \cdot 4 \%$ \\
\hline
\end{tabular}

CI, Confidence interval.

Number of studies for ELISA-based tests $(n=5)$, and for ICT-based tests $(n=3)$; four studies for sample timing and six studies for infection status.

Unless otherwise stated the $P$ value for heterogeneity between studies is $<0 \cdot 05$.

* Heterogeneity between studies was not significant $(P>0 \cdot 05)$ 
(a)
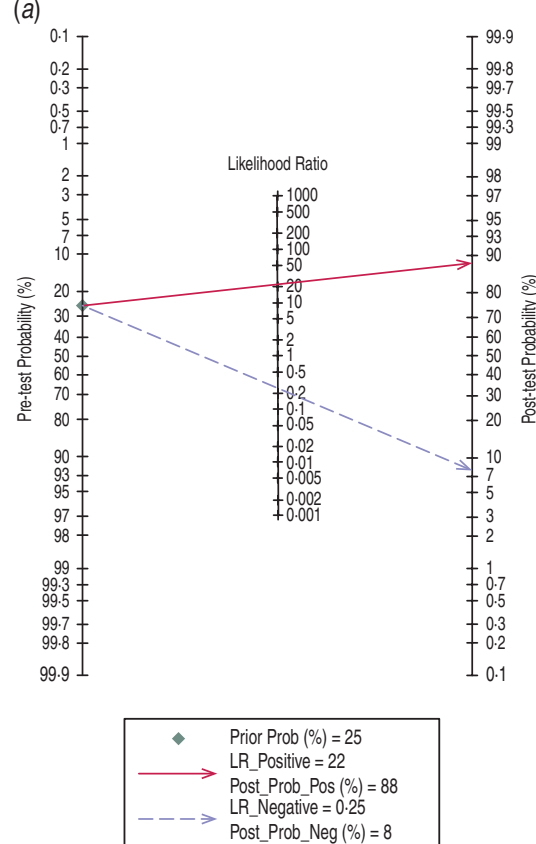

(b)
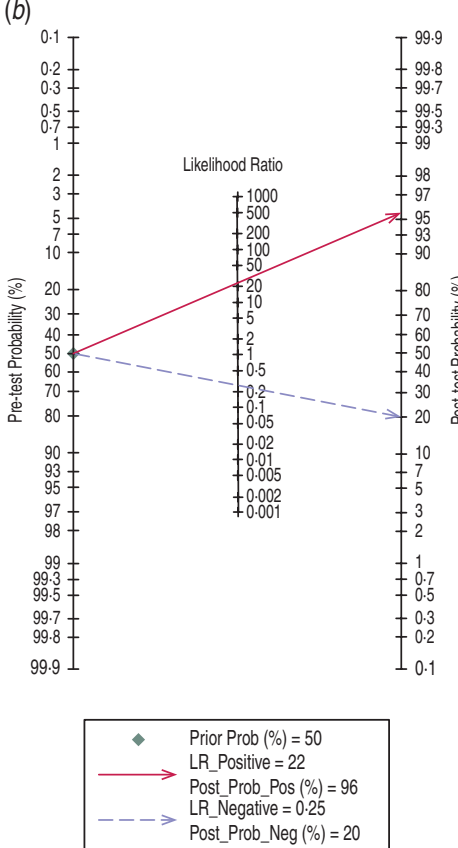

(c)
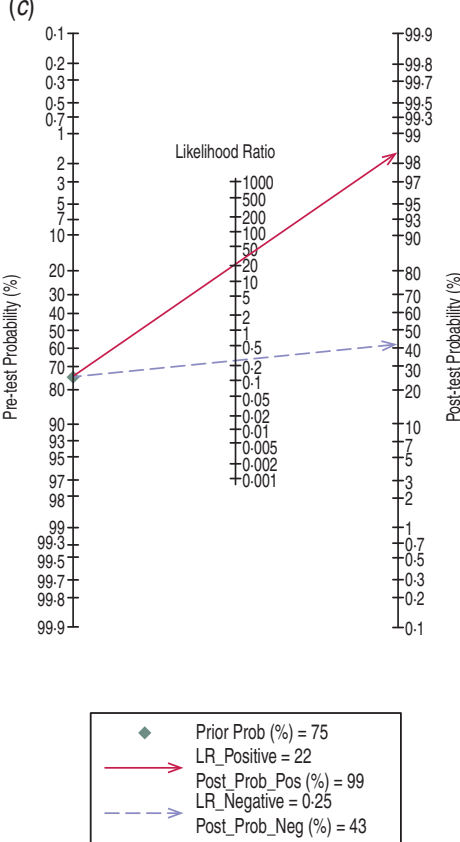

Fig. 5. Fagan's nomogram showing the post-test probabilities associated with IgA-based tests under different pre-test probabilities: (a) $25 \%$, (b) 50\%, (c) 75\%. Fagan's nomogram consists of three vertical axes, the first axis represents pretest probability, the middle axis represents the positive and negative likelihood ratios and the last axis represents post-test probability.

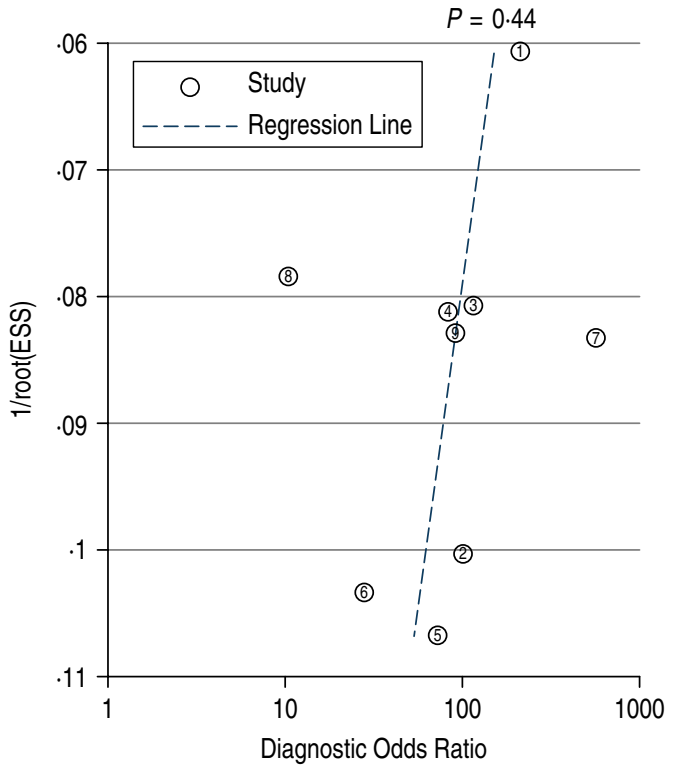

Fig. 6. Deeks' funnel plot for publication bias. Each circle represents an individual study and the dashed line represents the regression line. $P$ value $=0 \cdot 44$, suggesting no publication bias.

Significant heterogeneity was observed between the studies. Hence, subgroup analysis was performed on the basis of type of test, day of sample collection and immune status to identify the source of heterogeneity. A meta-regression analysis based on the year of publication and the sample size was also performed. The analysis revealed that type of the test and the day of sample collection could be possible sources of heterogeneity. The DENV serotype responsible for the infection, disease severity, ethnicity and geographical location of the study might also add to the heterogeneity observed in the study. Only three studies had data on serotypes while disease severity data was not available in all the studies except one $[10,11,26]$.

Based on the summary statistics, ELISA-based tests were more accurate in diagnosing dengue compared to ICT-based tests. If a patient had a negative test result based on ICT, the probability that the patient had dengue was $29 \%$, while for ELISA-based results it was $19 \%$. All the ELISA-based studies were based on IgA capture ELISA except the study by Yap et al. which utilized an antigen capture IgA ELISA $[8,9,16,17]$. The dOR for ACA ELISA was 564 and thus ACA-ELISA represents a promising test for diagnosis of dengue but needs evaluation in multiple prospective cohorts. Only three studies were available for meta-analysis of ICT-based tests. Although based on the summary statistics, the results of the ICT-based test were promising initially in a 
study with a dOR of 71 and LR - of 0.15 [19], results from subsequent studies with smaller sample sizes were not encouraging and further evaluation in larger prospective cohorts is required [20-23]. ICT-based tests had the advantage of providing point of care diagnosis within minutes without the requirement of costly equipment compared to the ELISA-based tests. However, since the reading is based on visual inspection, the ICT format has the weakness of subjective reading by the operator [29].

Irrespective of the type of test, IgA-based tests were more accurate in detecting dengue in the samples collected 4 days after symptom onset compared to the samples that were collected 4 days before. IgA-based tests had a greater accuracy in diagnosing secondary infections compared to primary infections. An earlier study reported that positivity for dengue-specific IgA was detected at about day 5 of onset of symptoms in primary infection while in secondary infections, positivity for $\operatorname{IgA}$ was detected around 4 days after symptom onset [7]. The results suggest that IgA-based tests could be of utility in settings where dengue is endemic and more secondary infections were occurring. It also indicated that the IgA-based tests would be more efficient in diagnosing dengue for the samples taken 4 days after symptom onset.

Prevalence rates of dengue vary according to geographical region. The Fagan plot analysis suggested that with an increase in the prevalence rate of dengue disease, post-test probability of positive IgA test results increased to more than $95 \%$ in dengue cases. However, the post-test probability of negative test results in dengue-positive cases also increased. This suggests that the proportion of false-negative $\operatorname{IgA}$ test results would be higher under the conditions of higher prevalence rates. In India, where dengue is endemic in most states, positivity rates for dengue in suspected cases ranged from $30 \%$ to $60 \%$ during different time periods [30]. The Fagan plot analysis revealed that even under a prevalence rate of $0.5,20 \%$ of false-negative results were expected in dengue cases. These results corroborated the finding that negative $\operatorname{IgA}$ test results should be confirmed with other dengue diagnostic tests.

Recent studies have reported that the combination of NS1 Ag and IgM antibody-based tests improve the sensitivity of serological tests [29]. Since IgA also appears in the body fluids during the same time period as IgM, the combination of NS1 Ag and IgA antibody-based tests can serve as an alternative. Although IgM- and IgA-based tests are equivalent in terms of sensitivity in detecting dengue [11, 22], IgA-based tests might have an advantage over IgM-based tests in secondary infections. NS1 Ag-based tests also had lower sensitivity during secondary infections [3-5]. Hence, the combination of NS1 Ag and IgA antibody-based tests might help in improving the diagnosis of dengue disease in countries where higher numbers of secondary infections occur. Prospective studies in different settings are needed to evaluate the diagnostic accuracy of combinations of IgM- and IgA-based tests with the NS1 Ag test.

In the present study, no publication bias was observed. However, the existence of publication bias cannot be excluded since the Deeks' funnel plot asymmetry test had low power when the included studies had significant heterogeneity [17]. Quality analysis of the studies included in the meta-analysis revealed that $33 \cdot 3 \%$ of the studies used samples from healthy controls. Using the samples from healthy controls for evaluation of diagnostics might exaggerate the diagnostic accuracy of the study. In $66.7 \%$ of the studies, it was unclear whether the reference test results were blinded to the people who performed the index tests. Another limitation of performing and interpreting meta-analysis of the diagnostic accuracy of tests for detecting dengue was the use of multiple reference tests by the study groups. In most of the excluded studies, testing of single serum samples by reference ELISAs (NS1 antigen ELISA, IgM capture ELISA, IgG capture ELISA) was used to define dengue cases. Details about disease severity was lacking in all the studies. Earlier studies have reported higher levels of denguespecific IgA in severe dengue cases [31, 32]. This calls for more prospective studies with data on disease severity, infection status, day of sampling and uniform criteria/ guidelines for the reference test to perform comparative evaluation of IgA-based and other antibody/antigenbased tests to diagnose dengue.

To conclude, the results of the present metaanalysis suggest that IgA-based assays have a moderate accuracy to detect dengue. The type of test, immune status and the day of sampling influenced the diagnostic accuracy of IgA-based tests. IgAbased tests can supplement other diagnostic tests and might be of use in settings of dengue endemicity with more secondary infections.

\section{DECLARATION OF INTEREST}

None.

\section{REFERENCES}

1. Shepard DS, Undurraga EA, Halasa YA. Economic and disease burden of dengue in Southeast Asia. PLoS Neglected Tropical Diseases 2013; 7: e2055. 
2. WHO. Comprehensive guidelines for prevention and control of dengue and degue hemorrhagic fever, 2nd edn. Regional Office for South-East Asia: World Health Organization, 2011, pp. 17-30.

3. Kosasih $\mathbf{H}$, et al. The diagnostic and prognostic value of dengue non-structural 1 antigen detection in a hyperendemic region in Indonesia. PLoS ONE 2013; 8: e80891.

4. da Costa VG, Marques-Silva AC, Moreli ML. A metaanalysis of the diagnostic accuracy of two commercial NS1 antigen ELISA tests for early dengue virus detection. PLoS ONE 2014; 9: e94655.

5. Acosta PO, et al. False-negative dengue cases in roraima, Brazil: an approach regarding the high number of negative results by NS1 ag kits. Revista do Instituto de Medicina Tropical de São Paulo 2014; 56: 447-450.

6. Peeling RW, et al. Evaluation of diagnostic tests: dengue. Nature Reviews Microbiology 2010; 8: S30-S38.

7. Vázquez $\mathbf{S}$, et al. Serological markers during dengue 3 primary and secondary infections. Journal of Clinical Virology 2005; 33: 132-137.

8. Talarmin A, et al. Immunoglobulin A-specific capture enzyme-linked immunosorbent assay for diagnosis of dengue fever. Journal of Clinical Microbiology 1998; 36: 1189-1192.

9. Yap G, Sil BK, Ng LC. Use of saliva for early dengue diagnosis. PLoS Neglected Tropical Diseases 2011; 5: e1046.

10. Tan YY, et al. Development of ASSURE Dengue IgA Rapid Test for the Detection of Anti-dengue IgA from Dengue Infected Patients. Journal of Global Infectious Diseases 2011; 3: 233-40.

11. De Decker S, et al. Evaluation of the diagnostic accuracy of a new dengue $\operatorname{IgA}$ capture assay (Platelia Dengue $\operatorname{IgA}$ Capture, Bio-Rad) for dengue infection detection. PLoS Neglected Tropical Diseases 2015; 9: e0003596.

12. WHO. Dengue guidelines for diagnosis, treatment, prevention and control: new edition. Geneva: World Health Organization, 2009.

13. Review Manager (RevMan). [Computer program]. Version 5.3. Copenhagen: The Nordic Cochrane Centre, The Cochrane Collaboration, 2014.

14. Whiting PF, et al. QUADAS-2: A revised tool for the quality assessment of diagnostic accuracy studies. Annals of Internal Medicine 2011; 155: 529-536.

15. Zamora J, et al. Meta-DiSc: a software for metaanalysis of test accuracy data. BMC Medical Research Methodology 2006; 6: 31 .

16. Fagan TJ. Nomogram for Bayes theorem [Letter]. New England Journal of Medicine 1975; 293: 257.

17. Deeks JJ, Macaskill P, Irwig L. The performance of tests of publication bias and other sample size effects in systematic reviews of diagnostic test accuracy was assessed. Journal of Clinical Epidemiology 2005; 58: 882-893.

18. Nawa M, et al. Evaluation of immunoglobulin A capture enzyme linked immunosorbent assay for sero diagnosis of dengue virus infection. Dengue Bulletin 2006; 30: 157-161.

19. Nawa M, et al. Immunoglobulin A antibody responses in dengue patients: a useful marker for serodiagnosis of dengue virus infection. Clinical and Diagnostic Laboratory Immunology 2005; 12: 1235-1237.

20. Matheus S, et al. Kinetics of dengue non-structural protein 1 antigen and $\operatorname{IgM}$ and $\operatorname{IgA}$ antibodies in capillary blood samples from confirmed dengue patients. American Journal of Tropical Medicine and Hygiene 2014; 90: 438-443.

21. Balmaseda A, et al. Diagnosis of dengue virus infection by detection of specific immunoglobulin $\mathrm{M}(\operatorname{IgM})$ and IgA antibodies in serum and saliva. Clinical and Diagnostic Laboratory Immunology 2003; 10: 317-322.

22. Balmaseda A, et al. Evaluation of immunological markers in serum, filter-paper blood spots, and saliva for dengue diagnosis and epidemiological studies. Journal of Clinical Virology 2008; 43: 287-291.

23. Groen $\mathbf{J}$, et al. Diagnostic value of dengue virus-specific $\mathrm{IgA}$ and $\operatorname{IgM}$ serum antibody detection. Microbes and Infection 1999; 1: 1085-1090.

24. Ahmed F, et al. Evaluation of ASSURE ${ }^{\circledR}$ Dengue $\operatorname{IgA}$ Rapid Test using dengue-positive and dengue-negative samples. Diagnostic Microbiology and Infectious Diseases 2010; 68: 339-344.

25. Sharmin R, et al. Dengue specific IgA in different body fluids: a prospective alternative for dengue diagnosis in resource-poor settings. Dengue Bulletin 2012; 36: 124-133.

26. de la Cruz Hernández SI, et al. Evaluation of a novel commercial rapid test for dengue diagnosis based on specific IgA detection. Diagnostic Microbiology and Infectious Diseases 2012; 72: 150-155.

27. Hasan SF, et al. To evaluate the efficacy and reliability of commonly available tests for diagnosis of dengue fever in children in Karachi. Pakistan Journal of Medical Research 2013; 52: 84-87.

28. Naz A, et al. Evaluation of efficacy of various immunochromatographic rapid tests for dengue diagnosis. Pakistan Journal of Medical Science 2014; 30: 166-171.

29. Blacksell SD. Commercial dengue rapid diagnostic tests for point-of-care application: recent evaluations and future needs? Journal of Biomedicine and Biotechnology 2012; 15: 1967

30. Cecilia D. Current status of dengue and chikungunya in India. WHO South-East Asia Journal of Public Health 2014; 3: 22-27.

31. De Rivera IL, et al. Humoral immune response of dengue hemorrhagic fever cases in children from Tegucigalpa, Honduras. American Journal of Tropical Medicine and Hygiene 2008; 79: 262-266.

32. Koraka P, et al. Kinetics of dengue virus-specific serum immunoglobulin classes and subclasses correlate with clinical outcome of infection. Journal of Clinical Microbiology 2001; 39: 4332-4338. 